\title{
ZnO Nanostructures on Electrospun Nanofibers by Atomic Layer Deposition/Hydrothermal Growth and Their Photocatalytic Activity
}

\author{
Fatma Kayaci, ${ }^{1,2}$ Sesha Vempati, ${ }^{* 1}$ Cagla Ozgit-Akgun, ${ }^{1,2}$ Necmi Biyikli ${ }^{1,2}$ and Tamer Uyar ${ }^{* * 1,2}$ \\ ${ }^{1}$ UNAM-National Nanotechnology Research Center, Bilkent University, Ankara, 06800, Turkey \\ ${ }^{2}$ Institute of Materials Science \& Nanotechnology, Bilkent University, Ankara, 06800, Turkey \\ Authors for correspondence: *svempati01@qub.ac.uk; **uyar@unam.bilkent.edu.tr
} \section{ABSTRACT}

A hierarchy of nanostructured- $\mathrm{ZnO}$ was fabricated on the electrospun nanofibers by atomic layer deposition (ALD) and hydrothermal growth, subsequently. Firstly, we produced poly(acrylonitrile) (PAN) nanofibers via electrospinning, then ALD process provided a highly uniform and conformal coating of polycrystalline $\mathrm{ZnO}$ with a precise control on the thickness (50 $\mathrm{nm}$ ). In the last step, this $\mathrm{ZnO}$ coating depicting dominant oxygen vacancies and significant grain boundaries was used as a seed on which single crystalline $\mathrm{ZnO}$ nanoneedles (average diameter and length of $\sim 25 \mathrm{~nm}$ and $\sim 600 \mathrm{~nm}$, respectively) with high optical quality were hydrothermally grown. The detailed morphological and structural studies were performed on the resulting nanofibers, and the photocatalytic activity (PCA) was tested with reference to the degradation of methylene blue. The results of PCA were discussed in conjunction with photoluminescence response. The nanoneedle structures supported the vectorial transport of photo-charge carriers, which is crucial for high catalytic activity. The enhanced PCA, structural stability and reusability of the PAN/ZnO nanoneedles indicated that this hierarchical structure is a potential candidate for waste water treatment.

\section{INTRODUCTION}

Development of novel materials with enhanced photocatalytic activity (PCA) along with stability is one of the intensely researched topics for water purification and waste treatment. The need for such research arises due to water pollution and ever increasing environmental issues threatening the human health severely [1-3]. Metal oxides such as $\mathrm{ZnO}$ in the structures of nanoparticles [4], nanorods [5, 6] and nanofibers [4] are widely studied for water purification purposes due to their well known PCA. On the other hand, electrospun polyacrylonitrile (PAN) nanofibers have been widely adopted in water filtration due to their unique properties including high surface area, nanoporous structure, low basis weight, easy permeability, good stability and chemical resistance [7-10]. Here we fabricated a hierarchy of nanostructured-ZnO depicting a synergy effect to enhance the PCA on electrospun PAN nanofibers using chemical vapor deposition and liquid phase deposition techniques, namely atomic layer deposition (ALD) and hydrothermal growth [11].

\section{EXPERIMENTAL DETAILS}

$\mathrm{N}, \mathrm{N}$-dimethylformamide (Pestanal, Riedel) was used as a solvent to prepare $12 \%(\mathrm{w} / \mathrm{v})$ PAN (Mw: 150,000, Scientific Polymer Products, Inc.) solution. For the electrospinning of the PAN solution; feed rate, applied voltage and tip-to-collector distance were $1 \mathrm{~mL} / \mathrm{h}, 15 \mathrm{kV}$ and 12 $\mathrm{cm}$, respectively. $\mathrm{ZnO}$ seed deposition on electrospun PAN nanofibers was carried out at $\sim 200$ 
${ }^{\circ} \mathrm{C}$ in a Savannah S100 ALD reactor (Cambridge Nanotech Inc.) using diethylzinc ( SigmaAldrich) and HPLC grade water as the zinc precursor and oxidant, respectively. $\mathrm{N}_{2}$ was used as a carrier gas at a flow rate of $\sim 20 \mathrm{sccm}$. 400 cycles were applied via exposure mode (a trademark of Ultratech/Cambridge Nanotech Inc.) in which dynamic vacuum was switched to static vacuum before each precursor pulse. For the growth of $\mathrm{ZnO}$ nanoneedles on $3.6 \mathrm{mg}$ of PAN/ZnO seed by hydrothermal process $\left(90^{\circ} \mathrm{C}, 5 \mathrm{~h}\right)$ in crucible, equimolar $(0.02 \mathrm{M})$ zinc acetate dihydrate $(\geq 98 \%$, Sigma-Aldrich) and hexamethylene tetramine ( $\geq 99 \%$, Alfa Aesar) were used. The morphology of the samples was studied using a scanning electron microscope (SEM, FEI - Quanta 200 FEG) with a nominal $5 \mathrm{~nm}$ of Au/Pd sputter coating. Transmission electron microscope (TEM, FEITecnai G2F30) images and selected area electron diffraction (SAED) pattern were also obtained. Photoluminescence (PL) measurements were performed using Horiba Scientific FL-1057 TCSPC at an excitation wavelength of $360 \mathrm{~nm}$. Methylene blue (MB, Sigma-Aldrich, certified by the Biological Stain Commission) was used as a model organic dye to test PCA of the samples. The nanofibrous mats (weight: $3.6 \mathrm{mg}$ ) were immersed in quartz cuvettes containing the MB solution $(18.8 \mu \mathrm{M})$. The cuvettes were exposed to UV light (300 W, Osram, UltraVitalux, sunlight simulation) placed at a distance of $\sim 15 \mathrm{~cm}$. Dye concentrations in the cuvettes were measured using a UV-Vis-NIR spectrophotometer (Varian Cary 5000) at regular time intervals. We have repeated the PCA experiment twice (i.e. $2^{\text {nd }}$ and $3^{\text {rd }}$ cycles) for PAN/ZnO needle sample $(\sim 3.3 \mathrm{mg})$ to determine the reusability versus performance. All the figures are reproduced with permission from Ref [11]

\section{DISCUSSION}

$\mathrm{ZnO}$ seed-coated PAN nanofibers were fabricated through electrospinning and ALD processes, on which $\mathrm{ZnO}$ nanoneedles were hydrothermally grown [11]. Schematic representations of the electrospinning and ALD processes, and fabrication procedure for the hierarchical $\mathrm{PAN} / \mathrm{ZnO}$ needle nanofiber are illustrated in figure 1.

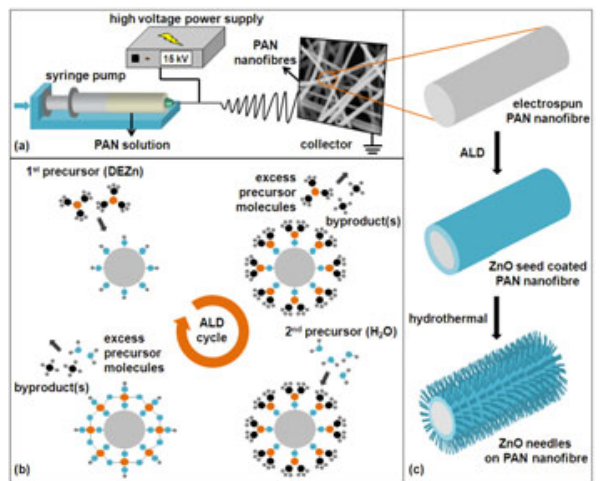

Figure 1. Schematic representations of the (a) electrospinning of PAN solution, (b) ALD of ZnO seed onto PAN nanofiber, and (c) fabrication procedure for hierarchical PAN/ZnO needle nanofiber. 
The representative SEM images of PAN nanofibers are given in figure 2(a), where the average fiber diameter (AFD) is estimated to be $\sim 655 \pm 135 \mathrm{~nm}$. After the ALD process, we have recorded the SEM images which are shown in figure 2(b) where the AFD is $\sim 715 \pm 125 \mathrm{~nm}$. AFD increased because of ALD coating, and moreover the fiber structure was stable during the ALD process. Subsequently, the hydrothermal method was employed to grow $\mathrm{ZnO}$ nanoneedles on the $\mathrm{ZnO}$ seed-coated PAN nanofibers. Straight $\mathrm{ZnO}$ nanoneedles covered the surface of the $\mathrm{ZnO}$ seed-coated PAN nanofibers, and no branching was observed, figure 2(c1-2). By analyzing the figure 2(c2), average diameter and length of the nanoneedles were determined as $\sim 25 \mathrm{~nm}$ and $\sim 600 \mathrm{~nm}$, respectively.
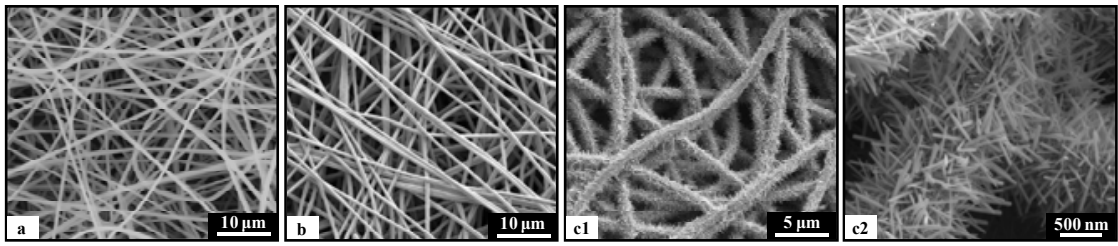

Figure 2. Representative SEM images of (a) pristine PAN, (b) PAN/ZnO seed, and (c1-2) $\mathrm{PAN} / \mathrm{ZnO}$ needle nanofibers at different magnifications.

Despite the relatively large surface area of the nanofibers, the conformal coating of $\mathrm{ZnO}$ with a uniform thickness $(\sim 50 \mathrm{~nm})$ was observed from the TEM image of PAN/ZnO seed nanofiber shown in figure 3(a1). For the high surface area substrates such as non-woven nanofiber mat, ALD is a well suitable technique as shown by us earlier [12,13]. The SAED pattern in figure 3(a2) reveals the polycrystalline nature of $\mathrm{ZnO}$ seed. The bright spots on the polycrystalline diffraction rings were observed due to the presence of well crystalline grains [13]. Moreover, high resolution TEM (HRTEM) and fast Fourier transform (FFT) images indicate the single crystalline nature of $\mathrm{ZnO}$ nanoneedles (figure 3(b1), (b2)). It is important to determine the growth direction of $\mathrm{ZnO}$; hence, the lattice spacing was measured as $\sim 0.525 \mathrm{~nm}$ corresponding to $c$-axis that is the preferential growth direction of the nanoneedles. Therefore polar planes of $\mathrm{ZnO}$ have shown to depict relatively higher PCA [14].
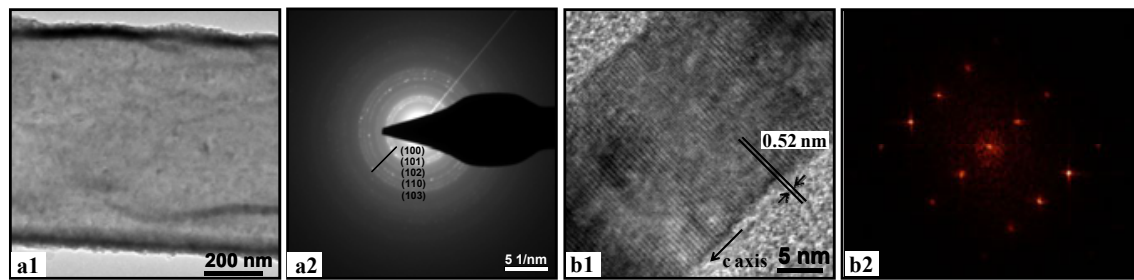

Figure 3. Representative (a1) TEM image and (a2) SAED pattern of PAN/ZnO seed nanofiber; (b1) HRTEM image and (b2) FFT image of $\mathrm{ZnO}$ needle.

We infered the information about surface defects from the PL spectra of nanofibers (figure 4a); such defects play a crucial role in determining the PCA of the material. Based on the literature, various crystal defects and the possible transitions with emission wavelengths are 
schematized in figure $4 \mathrm{~b}$. It is known that the typical excition emission band corresponding to the interband transition lies in the $\mathrm{UV}$ region for $\mathrm{ZnO}$, while the defect related emission is in the visible region [16-18]. PAN/ZnO needle has shown a clear peak in UV region, while PAN/ZnO seed did not show any clear excition emission peak. Violet emissions were broader, and not as prominent as green emission, which are related to zinc interstitials $\left(\mathrm{Zn}_{i}\right)$ and oxygen vacancies $\left(V_{\mathrm{O}}\right)$, respectively. PAN/ZnO seed has the predominant visible emission because of the large area of grain boundaries. For PAN/ZnO needle, a slight blue shift can be noticed in the center of the peak in the visible region of the spectrum due to its seed counterpart. Therefore $V_{\mathrm{O}}$ 's can create intermediate bands. Hence, the green emission is a combination of bulk grain region (BGR) and depletion region (DR). In BGR and in DR $V_{\mathrm{O}}{ }^{+} \rightarrow V_{\mathrm{O}}{ }^{*}$ and $V_{\mathrm{O}}{ }^{+} \rightarrow V_{\mathrm{O}}{ }^{++}$processes take place, respectively. $\mathrm{BGR}$ of $\mathrm{ZnO}$ is not accessible to the PCA, since it is in deep inside the lattice. However depletion layer within grain boundaries is extremely helpful for PCA. The intensity ratio of $\mathrm{UV}$ to visible emission is ten times higher for the $\mathrm{PAN} / \mathrm{ZnO}$ needle than $\mathrm{PAN} / \mathrm{ZnO}$ seed. This high ratio indicates higher optical quality of the PAN/ZnO needle sample.
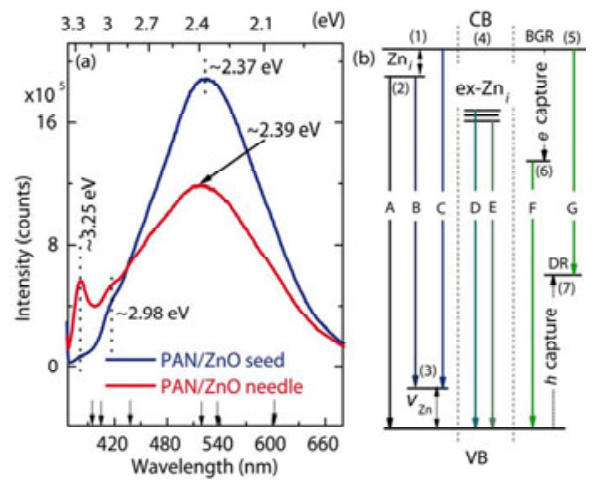

Figure 4. (a) PL spectra of PAN/ZnO seed and needle counterpart and (b) depicts various crystal defects and possible transitions [16]. The energetic location of each defect level (denoted by numerals) is obtained from the corresponding references (1) [19], (2) [20], (3) [21, 22], (4) [23], (5) $[17,18],(6)$ [24] and (7) [25]. The alphabets stand for emission wavelengths in nanometer, where $\mathrm{A}=395, \mathrm{~B}=437, \mathrm{C}=405, \mathrm{D}=440, \mathrm{E}=455, \mathrm{~F}=\sim 500$, and $\mathrm{G}=564$. $V_{\mathrm{Zn}}$ is located 0.30 $\mathrm{eV}$ above the $\mathrm{VB}$, while $\mathrm{Zn}_{i}$ is at $0.22 \mathrm{eV}$ below the $\mathrm{CB}$.

We have comparatively investigated the PCA of nanofibers (figure 5). Pristine MB solution data has shown a decay constant of $\sim 157 \mathrm{~min}$. On the other hand degradation rates clearly showed that the PCA was much higher for the needle sample ( 14.6 min) compared to seed one ( $\sim 113.1 \mathrm{~min})$. Under a suitable illumination $e$ and $h$ pairs are created in VB. Electrons can be excited from the VB to reach the $\mathrm{CB}$, leaving behind holes in the VB $[16,18]$. before these separated charges recombine, they can migrate to the surface of the semiconductor, then they can participate in the redox reactions to form hydroxyl radical $\left({ }^{\circ} \mathrm{OH}\right)$ that is the key for the PCA [26-28]. Interband transition in the PL spectrum showed that PCA is taking place at CB and VB in defect free nanoneedle. In the case of seed sample, there was no clear interband transition in PL, hence the PCA taking place at CB and VB was not dominant. In contrast, DR is well 
accessible for PCA for this sample. For PAN/ZnO needle case, significantly higher PCA was yielded through the combination of all these processes. As a result, $\mathrm{PAN} / \mathrm{ZnO}$ needle sample has shown higher PCA because of the higher surface area and higher crystal quality of the needlemorphology. Moreover catalytic activity occurs at surface defects on ALD seed and VB, and CB of nanoneedles. PCA experiment have been repeated twice for the PAN/ZnO needle, and the results showed almost no decay in the catalytic activity of this material when reused.
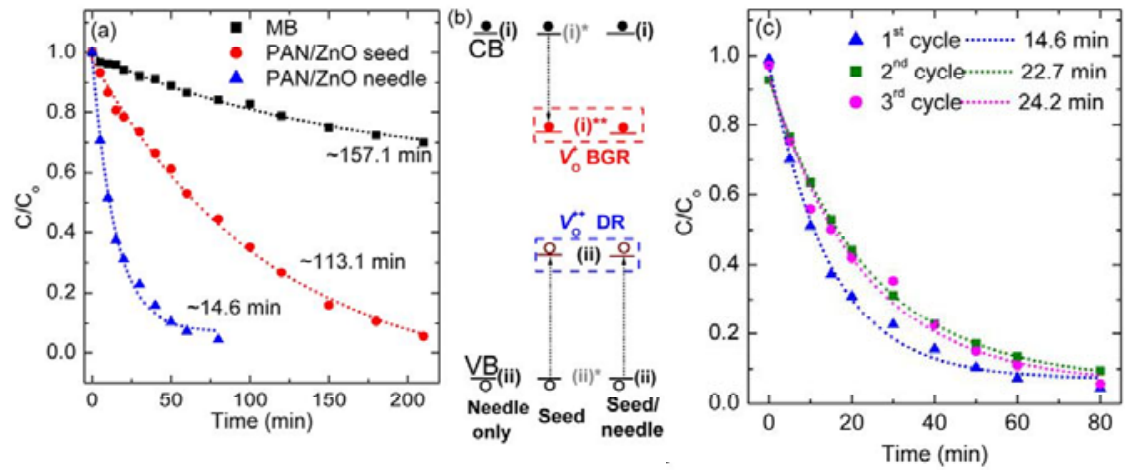

Figure 5. (a) Degradation rate of $M B$ in aqueous environment tested for pristine, in the presence of PAN nanofibers, PAN/ZnO seed and PAN/ZnO needle (1st cycle) cases, (b) plausible mechanism of photocatalysis involving oxygen vacancies, where (i) and (ii) stand for processes acceptor $\rightarrow$ acceptor ${ }^{-}$and donor $\rightarrow$ donor $^{+}$respectively, and (c) PCA of PAN/ZnO needle nanofibers for $1^{\text {st }}, 2^{\text {nd }}$ and $3^{\text {rd }}$ cycles.

\section{CONCLUSION}

Here hydrothermal growth of single crystal, needle like structures of $\mathrm{ZnO}$ was performed on the ALD seed coated electrospun PAN nanofibers. We have reported the results of an investigation on $\mathrm{ZnO}$ based photocatalyst. The PCA improvement was attributed to the collective effect, which enabled the active participation of defect state and the catalysis taking place at $\mathrm{CB}$, as well as VB. Moreover, the samples were subjected to recycling, and the PAN/ZnO needle depicted a comparable performance with the fresh sample. The performance, structural stability and reusability of these $\mathrm{ZnO}$ nanostructures make them potential candidates for waste water treatment with solar energy.

\section{ACKNOWLEDGMENTS}

S.V. thanks The Scientific \& Technological Research Council of Turkey (TUBITAK) (TUBITAK-BIDEB 2216, Research Fellowship Programme for Foreign Citizens) for postdoctoral fellowship. F.K. thanks TUBITAK-BIDEB for a PhD scholarship. N.B. thanks EU FP7-Marie Curie-IRG for funding NEMSmart (PIRG05-GA-2009-249196). T.U. thanks EU FP7-Marie Curie-IRG (NANOWEB, PIRG06-GA-2009-256428) and The Turkish Academy of Sciences - Outstanding Young Scientists Award Program (TUBA-GEBIP) for funding. Authors thank M. Guler for technical support for TEM analysis. 


\section{REFERENCES}

1. Q. Li, S. Mahendra, D. Y. Lyon, L. Brunet, M. V. Liga, D. Li and P. J. J. Alvarez, Water Res. 42, 4591 (2008).

2. F. Meng, S. R. Chae, A. Drews, M. Kraume, H. S. Shin and F. Yang, Water Res. 43, 1489 (2009).

3. M. M. Khin, A. S. Nair, V. J. Babu, R. Murugan and S. Ramakrishna, Energy Env. Sci. 5, 8075 (2012).

4. H. Liu, J. Yang, J. Liang, Y. Huang and C. Tang, J. Am. Ceram. Soc. 91, 1287 (2008).

5. A. Sugunan, V. K.Guduru, A. Uheida, M. S.Toprak and M. R. Muhammed, J. Am. Ceram. Soc. 93, 3740 (2010).

6. Z. Chang, Chem. Comm. 47, 4427 (2011).

7. N. Scharnagl, H. Buschatz, Desalination 139, 191 (2001).

8. S. Yang, Z. Liu, J. Membr. Sci. 222, 87 (2003).

9. L. Zhang, J. Luo, T.J. Menkhaus, H. Varadaraju, Y. Sun, H. Fong, J. Membr. Sci. 369, 499 (2011).

10. Y. Mei, C. Yao, K. Fan, X. Li, J. Membr. Sci. 417, 20 (2012).

11. F. Kayaci, S. Vempati, C. Ozgit-Akgun, N. Biyikli, T. Uyar, Applied Catalysis B: Environmental, http://dx.doi:10.1016/j.apcatb.2014.03.004, (2014).

12. F. Kayaci, C. Ozgit-Akgun, I. Donmez, N. Biyikli, T. Uyar, ACS Appl. Mater. Interfaces, 4, $6185(2012)$.

13. F. Kayaci, C. Ozgit-Akgun, N. Biyikli, T. Uyar, RSC Adv. 3, 6817 (2012).

14. J. Wang, P. Liu, X. Fu, Z. Li, W. Han, X. Wang, Langmuir, 251218 (2009).

15. S. Cho, J.-W. Jang, J.S. Lee, K.-H. Lee, Nanoscale, 4, 2066 (2012).

16. S. Vempati, J. Mitra, P. Dawson, Nanoscale Res. Lett. 7, 470 (2012).

17. J.D. Ye, S.L. Gu, F. Qin, S.M. Zhu, S.M. Liu, X. Zhou, W. Liu, L.Q. Hu, R. Zhang, Y. Shi, Y.D. Zheng, Appl. Phys. A: Mater. Sci. Process., 81, 759 (2005).

18. S. Vempati, S. Chirakkara, J. Mitra, P. Dawson, K.K. Nanda, S.B. Krupanidhi, Appl. Phys. Lett. 100, 162104 (2012).

19. C.H. Ahn, Y.Y. Kim, D.C. Kim, S.K. Mohanta, H.K. Cho J. Appl. Phys. 105, 013502 (2009).

20. E.G. Bylander, J. Appl. Phys. 49, 1188 (1978).

21. B. Lin, Z. Fu, Y. Jia, Appl. Phys. Lett. 79, 943 (2001).

22. P.S. Xu, Y.M. Sun, C.S. Shi, F.Q. Xu, H.B. Pan, Nucl. Instrum. Methods B 199286 (2003).

23. H. Zeng, G. Duan, Y. Li, S. Yang, X. Xu, W. Cai, Adv. Funct. Mater. 20, 561 (2010).

24. K. Vanheusden, W.L. Warren, C.H. Seager, D.R. Tallant, J.A. Voigt, B.E. Gnade, J. Appl. Phys. 79, 7983 (1996).

25. A.v. Dijken, E.A. Meulenkamp, D. Vanmaekelbergh, A. Meijerink, J. Lumin. 90, 123 (2000).

26. N. Daneshvar, D. Salari, A.R. Khataee, J. Photochem. Photobiol. A 162, 317 (2004).

27. R.W. Matthews, J. Catal. 97, 565 (1986).

28. Izumi, W.W. Dunn, K.O. Wilbourn, F.R.F. Fan, A.J. Bard, J. Phys. Chem. 84, 3207 (1980). 